\title{
Detection of a Pseudoaneurysm in the Knee Joint With Doppler Imaging After Blunt Trauma
}

\author{
Devrim Karaosmanoglu, MD, Musturay Karcaaltincaba, MD, \\ Okan Akhan, MD
}

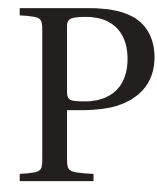

seudoaneurysms are secondary to the disruption of vascular wall continuity and are characterized by a cystic structure and swirling blood flow inside. They are commonly seen in contemporary medical practice secondary to increased interventional procedures and trauma. Several causative factors have been identified, and radiologists today must be concerned about their presence during daily routine practice. Although the inguinal area is the most common site because of arterial punctures of the femoral arteries, involvement of every vascular structure of the body has been reported. Treatment options depend on several factors, with size and location being the decisive ones. Pseudoaneurysms that are small and in atypical locations may be clinically occult and pose a diagnostic challenge. This report describes a pseudoaneurysm of the geniculate branch of the popliteal artery.

Abbreviations

$\mathrm{CT}$, computed tomographic

\section{Case Report}

An 8-year-old boy came to the emergency department 6 days after indirect trauma to his knee during a football match. During the initial examination, the chief symptoms of the patient were nonregressing soft tissue swelling and intense and unremitting pain, which became worse even with minor movement. The clinical examination was not notable except for intense knee pain, which was remarkably aggravated even with minor passive movement of the right knee, soft tissue swelling around the knee joint, and wide ecchymosis of the local skin.

Initial anteroposterior and lateral conventional radiographs revealed dislocation of the knee without bony fracture and prominent soft tissue edema. Before relocation of the knee joint, the emergency staff requested a sonographic examination because of concern about septic arthritis related to intense pain, swelling, and redness around the knee joint. The gray scale examination revealed a widespread hematoma between the muscle planes and a subcentimeter $(7 \times 8-\mathrm{mm})$ anechoic structure with swirling blood flow inside (Figure 1). The ensu- 


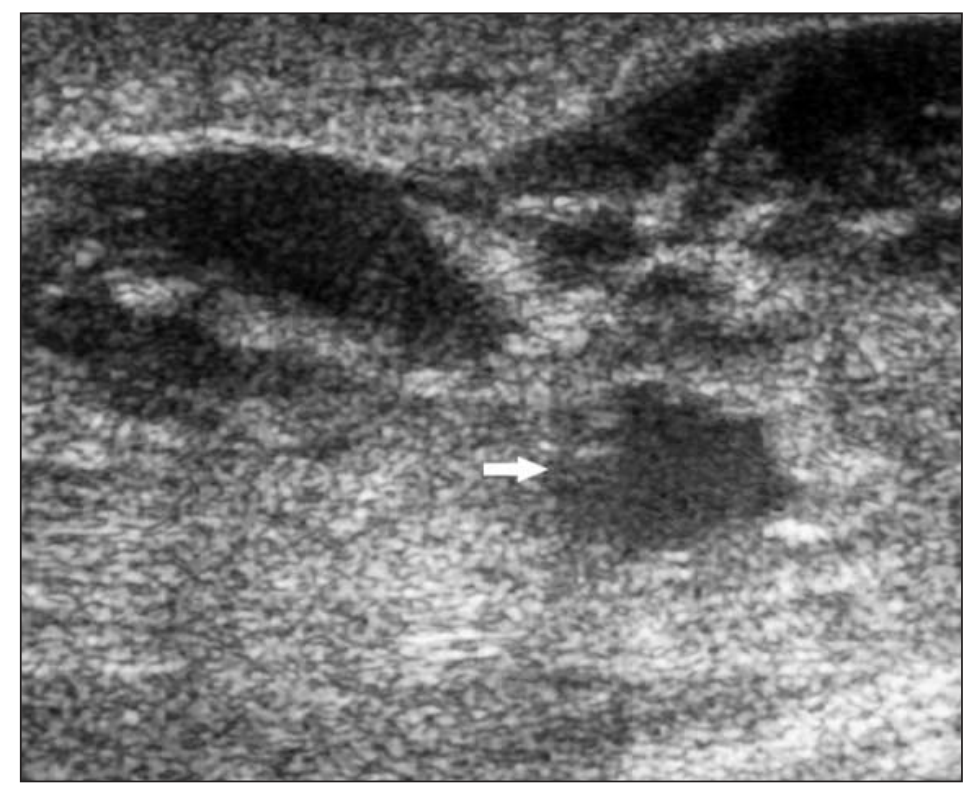

Figure 1. Real-time gray scale image shows an anechoic cystic structure with a swirling blood flow pattern inside (arrow).

ing color Doppler examination revealed the classic findings of a pseudoaneurysm (Figure 2A). Power Doppler examination more clearly depicted the feeding artery (Figure 2B). Spectral analysis also revealed a to-and-fro flow pattern. No evident thrombus was seen inside the pseudoaneurysm sac. The continuation of the flow with the popliteal artery could not be shown. Popliteal anterior tibial arteries and the tibioperoneal trunk and its runoff branches were patent, with triphasic flow documented on spectral examination. The popliteal vein and its major tributaries were also patent and fully compressible.

With the leading diagnosis of a traumatic pseudoaneurysm, the patient was referred for a computed tomographic (CT) angiographic study to rule out a possible additional vascular injury. The CT angiography confirmed the sonographic findings, revealing a subcentimeter pseudoaneurysm with no visible feeding artery; later, it was presumed to be supplied by a superolateral geniculate branch of the popliteal artery (Figure 3). The major arterial and vascular structures of the field were also found to be patent. A widespread hematoma and dislocation of the knee joint (depicted by the sagittally reformatted images) were among the other ancillary CT findings. After the CT examination, the patient underwent closed knee relocation under general anesthesia by the orthopedic surgeons.

\section{Discussion}

Pseudoaneurysms generally arise from a disruption in the integrity of the arterial wall secondary to various causes, including inflammation, trauma, and interventional procedures. ${ }^{1}$ Under the continuous influence of sustained arterial pressure, blood dissects into the neighboring soft tis-

Figure 2. A, Color Doppler image of the knee joint reveals a typical to-and-fro pattern of the pseudoaneurysm. The arrow indicates the feeding vessel. B, Power Doppler image of the knee joint. The feeding vessel can be seen more clearly (arrow).

A

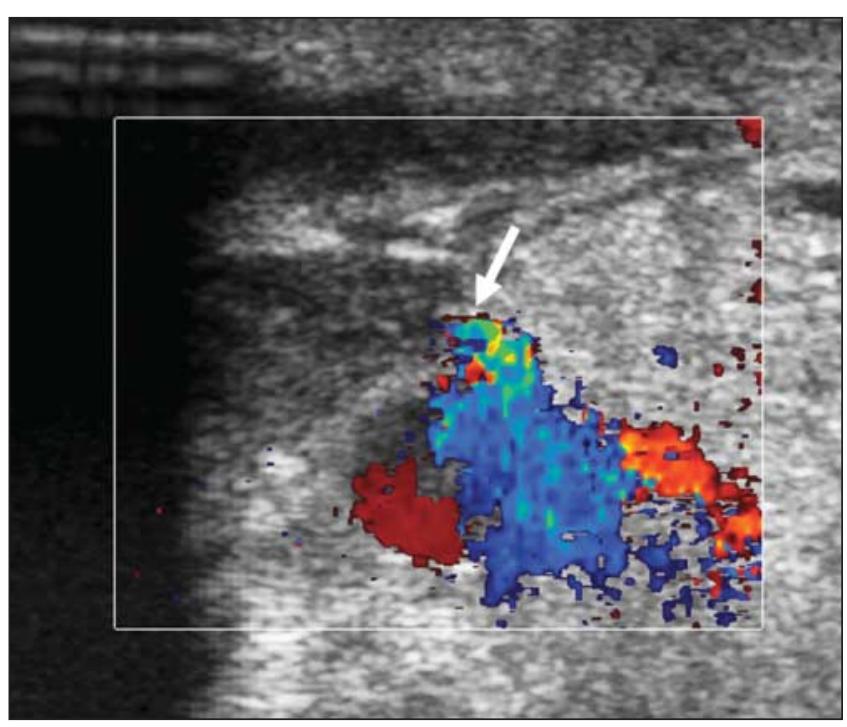

B

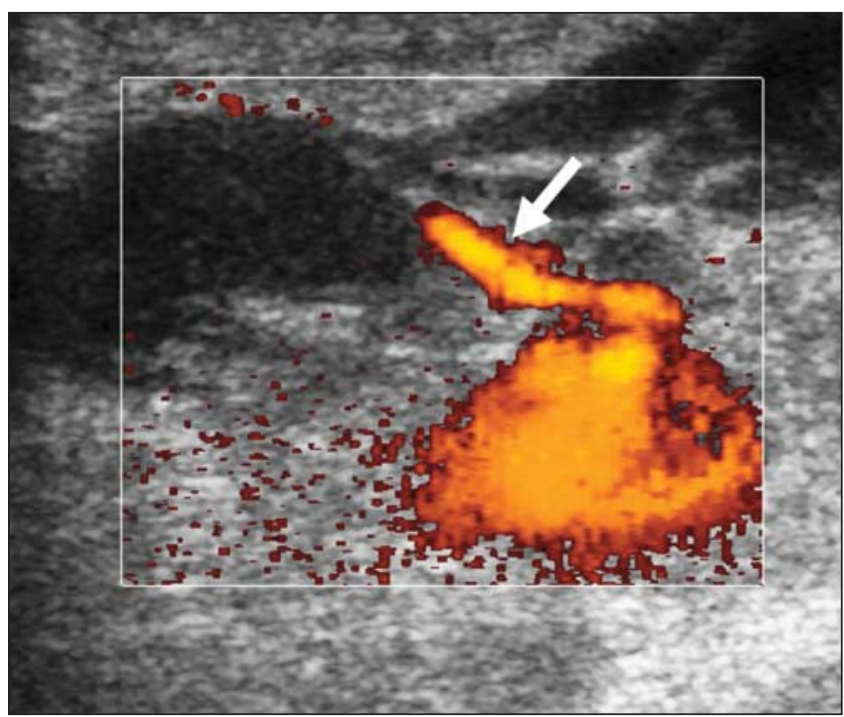


sue planes and forms a perfused sac that communicates with the arterial lumen. ${ }^{2}$ The perfused sac is contained by the media or adventitia or simply by soft tissue structures surrounding the injured vessel. Pseudoaneurysms have been reported in several areas of the body, including neck, limb, and visceral arteries.

Pseudoaneurysms of the geniculate arteries are rare and have generally been reported in the orthopedic literature after surgical interventions of the knee. ${ }^{3}$ Blunt and penetrating traumas to the knee may cause serious vascular injuries with potentially devastating consequences. Popliteal arterial injuries are not rare and may necessitate meticulous and relevant imaging and intervention. Sonography, particularly color Doppler imaging, is one of the best imaging modalities for the detection of pseudoaneurysms in these circumstances. It is easy to perform and inexpensive, obviates the use of iodinated contrast agents, and is radiation free. Sonography may also be used in selected cases for the treatment of pseudoaneurysms with considerable success. ${ }^{2}$

The previously reported cases of pseudoaneurysms of the geniculate arteries resulted from surgical interventions. The generally presumed mechanisms were periosteal elevation and hamstring tendon harvesting. ${ }^{4}$ Uncommon anatomic variations of the arterial tree may also be one reason for vascular injury during surgery.

To our knowledge, no pseudoaneurysm of the geniculate arteries after blunt knee trauma has been reported previously. Pseudoaneurysms must be assumed in this clinical circumstance after blunt trauma, even if they are rare. Additional diagnostic modalities must be used rapidly in cases of giant pseudoaneurysms. With these modalities, critical information regarding bony abnormalities, such as joint dislocation in our case, may be provided to the medical staff. With this case, we tried to emphasize the role of sonography, particularly color Doppler imaging, in the evaluation of patients with unexpected knee problems after surgical interventions and trauma. Examinations must be done with the maximum possible care, and color Doppler imaging should be used in cases of suspected pseudoaneurysms to provide important clinical data to both clinicians and interventional radiologists so that prompt therapeutic strategies may be considered, thereby preventing potentially devastating complications. ${ }^{5}$

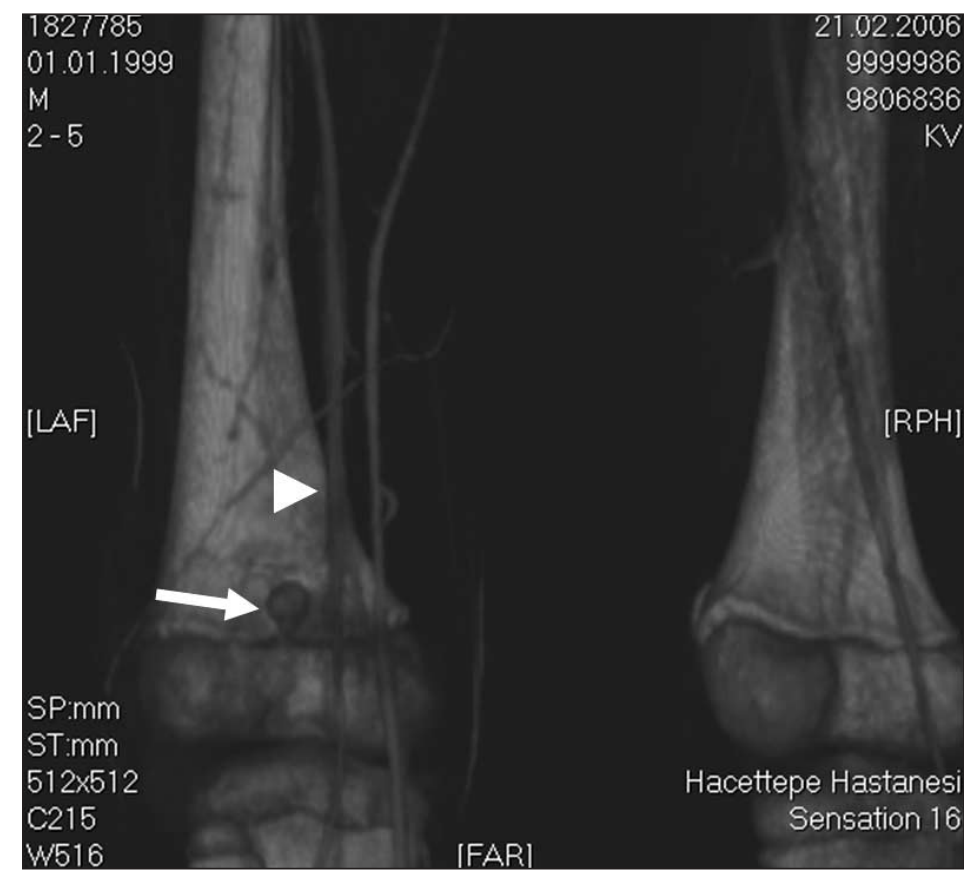

Figure 3. Computed tomographic angiographic image shows the pseudoaneurysm (arrow) in the superolateral portion of the knee in close proximity to the popliteal artery (arrowhead). Dislocation of the knee joint was also observed on sagittally reformatted images (not shown).

\section{References}

1. Schwartz LB, Clark ET, Gewertz BL. Anastomotic and other pseudoaneurysms. In: Rutherford RB (ed). Vascular surgery. 5th ed. Philadelphia, PA: WB Saunders Co; 2000:752-763.

2. Saad NE, Saad WE, Davies MG, Waldman DL, Fultz PJ, Rubens DJ. Pseudoaneurysms and the role of minimally invasive techniques in their management. Radiographics 2005; 1(suppl):S173-S189.

3. Milankov M, Miljkovic N, Stankovic M. Pseudoaneurysm of the medial inferior genicular artery following anterior cruciate ligament reconstruction with hamstring tendon autograft. Knee 2006; 13:170-171.

4. Evans JD, Boer MT, Mayor P, Rees D, Guy A. Pseudoaneurysm of the medial inferior genicular artery following anterior cruciate ligament reconstruction. Ann R Coll Surg Engl 2000; 82:182-184.

5. Bapuraj JR, Thingnam S, Rajak CL. Popliteal artery pseudoaneurysm following penetrating trauma. Australas Radiol 1999; 43:116-118. 